\title{
Multi-Point Touch Input Method for Korean Text Entry
}

\section{Heesook Shin}

Wearable Computing Research Team

Electronics and Telecommunications Research Institute 161, Gajeongno, Yuseong-gu, Daejeon, Korea

hsshin8@etri.re.kr

\section{Woohun Lee}

Design Media Lab.

Korea Advanced Institute of Science and Technology

335 Gwahangno, Yuseong-gu, Deajeon, Korea

woohun.lee@kaist.ac.kr

\section{Geehyuk Lee}

Human Computer Interaction Lab.

Information and Communications University

119, Munjiro, Yuseong-gu, Daejeon, Korea

geehyuk@icu.ac.kr

\section{Ilyeon Cho}

Wearable Computing Research Team

Electronics and Telecommunications Research Institute

161, Gajeongno, Yuseong-gu, Daejeon, Korea

iycho@etri.re.kr

Copyright is held by the author/owner(s).

CHI 2009, April 4-9, 2009, Boston, MA, USA.

ACM 978-1-60558-247-4/09/04.

\begin{abstract}
Multi-touch interfaces are becoming popular as a new input means for the various applications. In this paper, we suggest a new Korean text entry method using a multi-touch interface called MPT (Multi-Point Touch) input method. We conducted a text entry performance test comprising 4 sessions for 10 participants, and compared the result with an existing commercial SPT (Single-Point Touch) input method. The experimental results show that the entry speed of MPT was slower than that of SPT method in the initial session. However, the entry speed of MPT input method was improved more rapidly than the speed of SPT method as sessions were proceeded. We observed a statistically significant learning effect from the result of MPT method.

Moreover, we found no significant difference between the task loads of SPT and MPT input methods.
\end{abstract}

\section{Keywords}

multi-touch, text entry

\section{ACM Classification Keywords}

H.5.2. Information interfaces and presentation (e.g., $\mathrm{HCI}$ ): user interfaces, theory and methods. 


\section{Introduction}

As multi-touch sensing technology evolves, commercial devices with multi-touch functionality are being introduced such as 'Surface' from Microsoft and 'iPhone' from Apple. Multi-touch interfaces are widely studied as a novel, intuitive and natural input method. However, little is known about a multi-touch interface as a text entry method, which motivated this research. In this paper, we propose a new Korean text entry method using a multi-touch interface, and discuss the merits and limitations of the method.
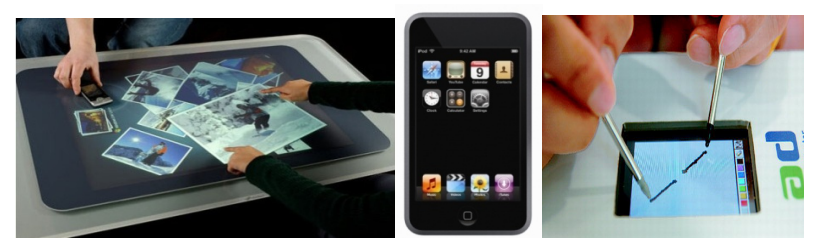

Figure 1. Computing devices with touch screen

\section{Related Works}

Several studies have been made on Korean text entry methods using a single touch point. Many of these studies use QWERTY keyboard layout, 3x4 button layouts or others in small devices. Some of them provide additional functions such as dynamic layout, visual assist, and so on. Some examples of single-touch Korean text entry methods are illustrated in Figure 2. When a consonant button is pressed, 'MOAkey' from Samsung shows additional buttons for inputting a vowel as illustrated in Figure 2a. This method has an advantage when a vowel often follows a consonant, which is true for Korean language. Examples in Figure
$2 b$ and $2 c$ show magnified-view functions [1]. Some text entry methods use the movement of a touch point. The method in Figure $2 d$ uses a vertical drag and a horizontal drag as commands for a character input [2].

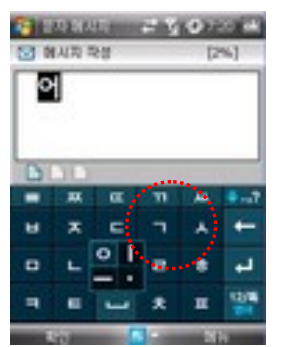

(a) MOAkey by Samsung

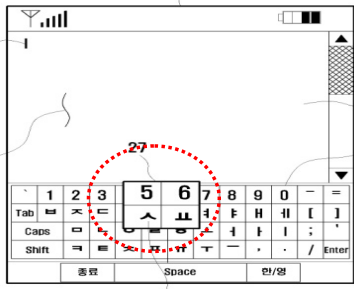

(c) Patent example I [1]

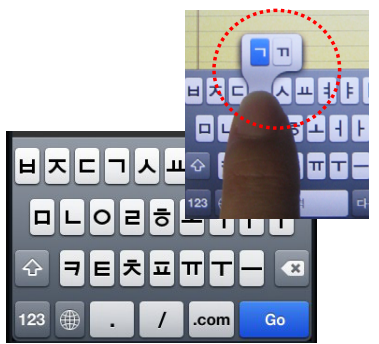

(b) Soft Keyboard in iPod Touch
Figure 2. Korean text entry methods in mobile devices with a touch screen

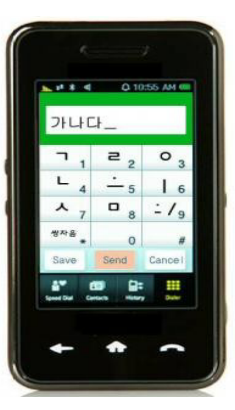

(d) Patent example II [2] 


\section{Multi-Point Touch Input Method for Korean \\ Text Entry}

Previous Korean text entry methods for touch screen devices are based on a single touch interface. In this section, we introduce MPT (Multi-Point Touch) input method based on multiple touch points shown in Table 1.

The left side of the table illustrates an existing commercial SPT (Single-Point Touch) input method and the right side illustrates the suggested MPT input method. The button configuration is assumed to be equal as in Figure 3.

If a user wants to input the Korean consonant ' 77 ', she has to touch the ' $\neg 77^{\prime}$ button with a finger two times using the SPT input method. However, if the MPT method is used, the ' $\neg 7$ ' button is touched only once with two fingers. In the case of ' $\|^{\prime}$ ', a user has to touch the ' ' button two times and the 'I ' button two times using the SPT method. But with the MPT method a user can enter the same character by touching the '.' button and the 'I ' button with two finger points. Using the

SPT input method, buttons are touched with one finger point only. But using the MPT input method, buttons can be touched with one finger point or two finger points [3][4].

The MPT method decreases the KSPC (keystrokes per character) but is expected to increase cognitive workload.

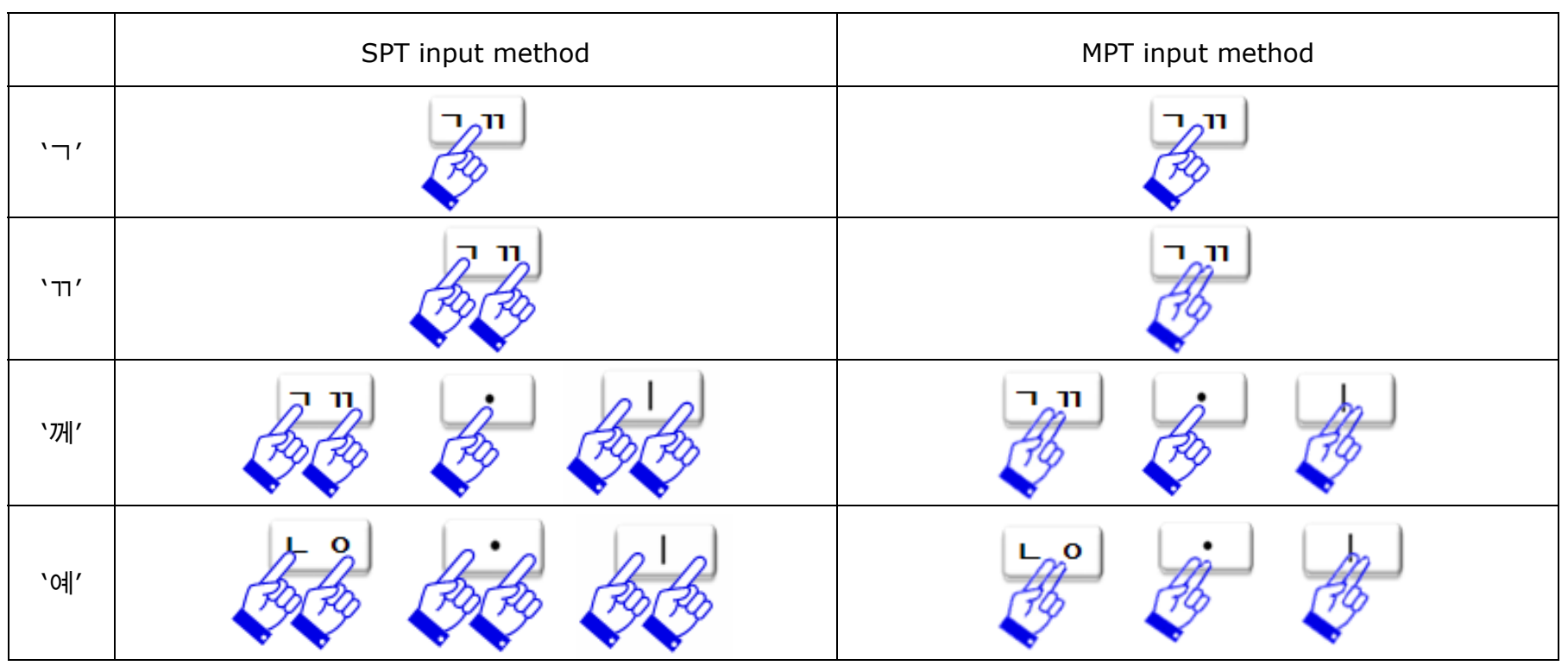

Table 1. SPT input method vs. MPT input method 


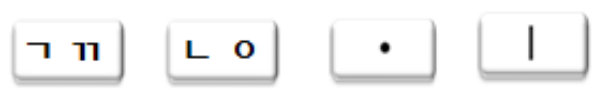

Figure 3. A button configuration with consonants and vowels

\section{Usability Test}

We conducted a quick-and-dirty usability test that compares the proposed MPT input method with the SPT input method. In both cases we used the 'CheonJiIn' keyboard layout shown in Figure $4 a$.

\section{Method}

We adopted the MacKenzie's Paper Mockups for the rapid evaluation of the MPT method since the paper mockups methodology was shown to be useful as a quick and efficient means to empirically test soft keyboard layouts [5]. In our test, we used iPod Touch devices displaying the image of a keyboard layout in order to offer closer similarity to the real environment. We covered the device with a transparent acrylic board to disable touch sensing as shown in Figure $4 a$.

We prepared 1100 test sentences from a commercial Korean typing practice sentences consisting of proverbs, gnomes, designations, and others. Each sentence comprises between 9 and 34 characters including blanks. As shown in Figure 4b, we used a program which selects a random sentence from the 1100 test sentences. Text entry time was calculated by measuring the start and end times of text inputs.
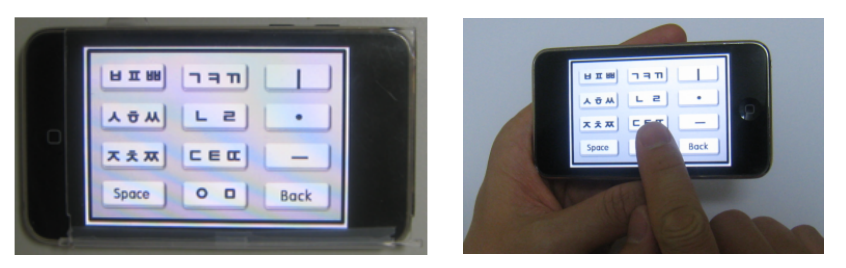

(a) An image-based paper mockup

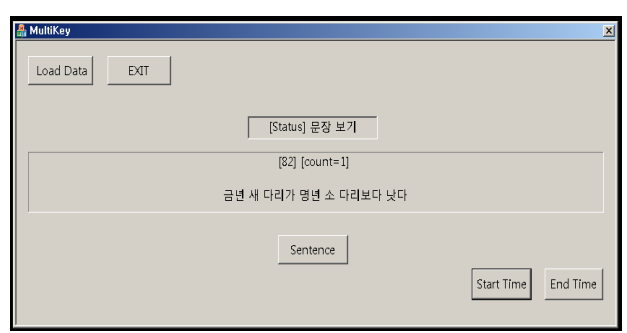

(b) A program to generate sentences and measure entry time

Figure 4. The usability test environment

\section{Participants}

We recruited 10 unpaid participants among college students and institute researchers with ages between 20 and 40 . Four of them were female and the average age of all the participants was $31.6(\mathrm{STD}=4.53)$. All participants were right-handed. Six participants were familiar with the 'CheonJiIn' Korean text entry method. We tried to select participants with a similar text entry skill in mobile devices. 


\section{Procedure}

Five participants ( 2 female, 3 male) were requested to use the MPT input method and the others were requested to use the SPT input method. All had an equivalent test environment. They entered displayed sentences by touching button images.

The user experiment was organized by 4 sessions. Text entry speeds for 15 sentences were recorded in each session. In the first session, the description for the input method was offered for 5 minutes and 5 exercise sentences were given for practice. In other sessions, participants were given two exercise sentences before the main trial was started.

Participants were requested to press the 'SPACE' button before and after entering a sentence as quickly as possible. Time between the two 'SPACE's was measured and was used to calculate an entry speed for each sentence [6]. We did not measure an error rate and just asked them to enter the sentences trying not to make mistakes. We allowed them to correct their typos after touching 'BACK' button.

After the final session, we asked participants to fill out the NASA-TLX sheet for work load measurement [7].

\section{Results}

The average entry speed of MPT input method was 52.45CPM (Character Per Minute) $(S T D=6.99)$ and that of SPT was 52.33CPM (STD=2.52) for the total of 60 trials. Figure 5 shows entry speeds in each session. The CPM results obtained are expected to be a little faster than the actual entry speeds because we allowed erroneous inputs.

As Figure 5 shows, participants' entry speed increased with practice for both SPT and MPT methods. The entry speed of MPT method was measured slower than that of SPT method in the initial session. However, the entry speed of MPT input method improved more rapidly than that of SPT method as sessions were repeated. In the case of MPT input method, the CPM of final session showed a $25.59 \%$ increase over that of the first session. However, in the case of SPT method, it showed only a $10.09 \%$ increase.

We also found that there was a statistically significant difference between trials ( $F(N=5$, Chi-Square $=12.12$, $\mathrm{df}=3, \mathrm{p}=0.007)$ ) in the case of MPT input method. From the result, the session effect of our MPT method showed significant improvement with practice.

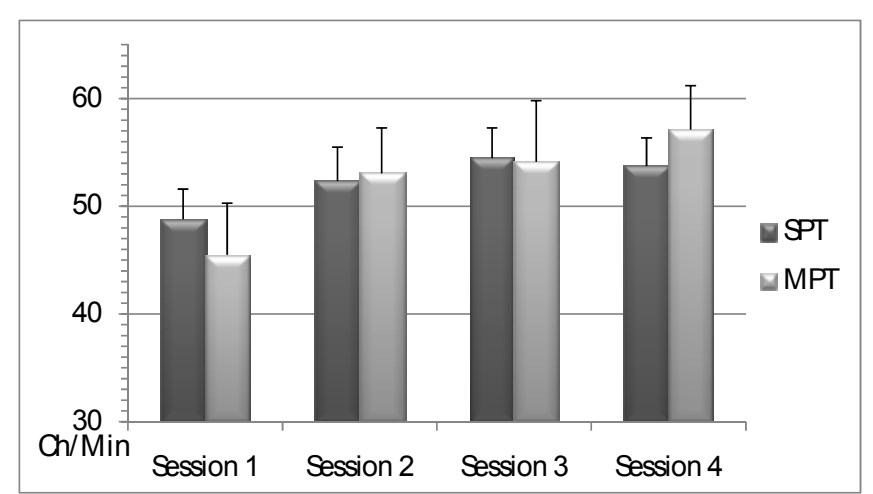

Figure 5. Text Entry Speed by Methods and Sessions

We evaluated the task-load of two methods using the NASA-TLX test. The result is shown in Figure 6 . The average task-load of MPT method was 320.4

$(\mathrm{STD}=53.34)$, for SPT method 315.12(STD=68.85).

From the Mann-Whitney $U$ test, $p$ value showed 0.754 $(U=11, W=26, Z=-0.313, p(2$-tailed $)=0.754)$. 
The result clearly showed that there was no significant difference between task-load of SPT method and MPT method.

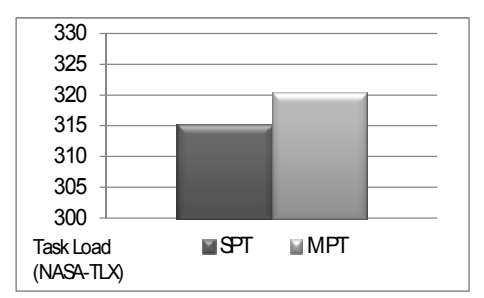

Figure 6. Comparison of the Task-load

\section{Conclusion}

We predicted that the MPT method would decrease the KSPC (Keystrokes per character) and increase the cognitive workload. From the quick-and-dirty usability test with 10 participants (between-subject) for 4 sessions, we could not find any significant difference between text entry speed of MPT input method and SPT input method. But the MPT method showed the obvious learning effect from the Friedman test.

Contrary to our expectation, the result indicated that there was no significant difference of the workload, which is occurred from the complicated decision-making process for the text entry.

In conclusion, we proposed and evaluated a new multipoint touch input method which can improve the efficiency of existing text entry method using single point touch.
For the future research, we are going to implement a realistic environment and conduct longer term usability test for more reliable evaluation. We also think the MPT method would show a significant improvement in text entry speed if we have a long term usability test. From this work we would be able to design more efficient keyboard layout for multiple point touch interface and improve our MPT input method.

\section{Acknowledgements}

This work was supported by the IT R\&D program of MKE/IITA, [2008-S001-08MC3810, Wearable Personal Companion].

\section{Reference}

[1] Device for Input Korean Character in Electric Appliances with Touch Screens, Korea Patent, Publication Number 2008-0072810

[2] Method for Inputting Character in Terminal Having Touch Screen, Korea Patent, Publication Number 20080029028

[3] Digital Hangeul Museum.

http://www.hangeulmuseum.org

[4] Hangeul Conference. http://www.hangeul.or.kr

[5] Mackenzie, I. S., and Read, J. C. Using Paper Mockups for Evaluating Soft Keyboard Layouts. In Proceedings of CASCON 2007, 98-108.

[6] MacKenzie, I. S., Note on Calculating Text Entry Speed. http://www.yorku.ca/mack/RN-TextEntrySpeed.html

[7] Seoktae Kim, Minjung Sohn, Jinhee Pak, Woohun Lee. One-key keyboard: A Very Small QWERT Keyboard Supporting Text Entry for Wearable Computing. In Proceedings of the 18th Australia conference on CHI 2006, 305-308. 